\title{
Vita in famiglia e responsabilità genitoriali nel lockdown della primavera 2020. Racconti e rappresentazioni di alunne/i di scuola secondaria di primo grado di Bologna
}

\author{
Stefania Lorenzini ${ }^{1}$
}

\begin{abstract}
Il contributo analizza i principali contenuti di brevi componimenti realizzati da ragazze e ragazzi di scuola secondaria di primo grado di Bologna chiamati a esprimere in poche righe scritte l'esperienza in corso: il cosiddetto lockdown, che tutti ha coinvolto e condizionato dal marzo 2020 in poi. L'attenzione è posta in particolare sui racconti centrati sulla vita familiare nel periodo di confinamento, su interrogativi, bisogni, preoccupazioni, paure, emozioni, criticità e risorse che le/gli alunne/i hanno esposto nei loro scritti. E sulle responsabilità educative che essi sollecitano.
\end{abstract}

Parole chiave: lockdown, alunne/i secondaria di primo grado, componimenti scritti, vita familiare, pandemia.

\begin{abstract}
The paper analyses the main contents of short essays written by girls and boys of secondary school in Bologna who were asked to express in a few written lines the ongoing experience: the so-called lockdown that involved and conditioned everyone from March 2020 onwards. Particular attention is paid to the stories centred on family life during the period of confinement, on the questions, needs, worries, fears, emotions, critical points and resources that the pupils have set out in their writings. And on the educational responsibilities they call for.
\end{abstract}

Keywords: lockdown, secondary school pupils, written essays, family life, pandemic.

${ }^{1}$ Professoressa associata e Docente di Pedagogia interculturale presso il Dipartimento di Scienze dell'educazione dell'Università degli Studi di Bologna. 


\section{Introduzione}

A partire dal marzo 2020, nel periodo di lockdown vissuto in Italia e a livello globale, tra le molteplici implicazioni della pandemia da Covid-19, è venuto a mancare anche il fondamentale ruolo svolto dalla scuola in presenza che, come evidenzia Oliverio Ferraris (cfr. cit. in Marrocco, 2020), per i ragazzi, anche per quelli che si dichiarano più "svogliati", rimane una "casa": una seconda famiglia, cruciale nell'età evolutiva. Cruciale in quanto luogo di incontro con un mondo più vario e differente da quello del nucleo familiare propriamente detto; luogo delle amicizie, delle alleanze e, in genere, luogo nel quale anche l'apprendimento avviene più agevolmente, grazie alla presenza degli insegnanti, agli stimoli provenienti dalle/dai compagne/i di classe, alla sensazione di "fare gruppo" e all'esperienza di stare insieme. Se «per crescere c'è bisogno di sentirsi posizionati socialmente» (Ibidem, passim), in mancanza dell'esperienza scolastica in presenza, la responsabilità di indicare ai più giovani una linea da seguire ricade ancora di più sulla famiglia.

In fase di confinamento entro gli spazi domestici, la famiglia - anche per coloro che come gli adolescenti stanno compiendo sempre più ampi passi verso l'autonomia e l'emancipazione fisica e psicologica da essa diviene l'unico ambiente relazionale e spazio concreto della vita quotidiana. Acquisendo una centralità ulteriore, se non totalizzante. All'interno delle famiglie ci si è trovati così a dover affrontare nuove esigenze e ad assumere ulteriori ruoli legati alla presenza continuativa dei figli a casa, allo svolgimento di tutte le attività scolastiche e della didattica a distanza, negli stessi ambienti in cui più fratelli/sorelle e adulti nelle loro attività lavorative hanno dovuto condividere una drastica riorganizzazione spaziotemporale della vita quotidiana. In certi casi, si è dovuto far fronte a difficoltà economiche legate alla perdita del lavoro in una fase di crisi.

In più, è intuibile come gli effetti su bambine/i e ragazzi/e di un lockdown causato da una pandemia dipendano in buona misura proprio dalle caratteristiche del contesto familiare: da atteggiamenti genitoriali ansiosi e spaventati, oppure di scarso riconoscimento della serietà dei pericoli in corso; dalla disponibilità a lasciare esprimere nuovi interrogativi, paure, dubbi, e dalla capacità di cercare e offrire spiegazioni, accogliere e sostenere emozioni; dall'abilità nel donare equilibrio alle relazioni ancora più strette e forzate entro spazi delimitati e per tempi protratti; dall'abilità nel riorganizzare la quotidianità, ma anche nel riprogettare l'immediato futuro senza perdere di vista fiducia e possibilità di ripresa, pur se nell'incertezza dei modi e dei tempi. Si pone con evidenza anche 
come le problematiche e le risorse materiali, educative, affettive e relazionali interne alla famiglia e già presenti prima della circostanza emergenziale carica di complessità, non possano che incidere in modi diversi su come la stessa può essere affrontata.

Alcune tra le ricerche svolte nella primavera 2020 mostrano dati che aiutano a comprendere come l'emergenza abbia impattato sul singolo, sulle coppie e sulle famiglie nella gestione dello stress, nella conciliazione tra i carichi di cura familiare e impegno lavorativo (laddove mantenuto). Questo è il caso del lavoro COVID-19 e conciliazione familiare e lavorativa: quali sfide e risorse per le famiglie italiane?, realizzato dal Centro di Ateneo Studi e Ricerche sulla Famiglia dell'Università Cattolica del Sacro Cuore di Milano. La ricerca ha coinvolto 1.400 intervistati di cui il $73 \%$ donne, in media di età superiore ai 45 anni $(56 \%)$, coniugati $(64 \%)$, conviventi $(10 \%)$, residenti nell'Italia nord-occidentale $(63,4 \%)$, con un livello sociale e di istruzione medio-alto (laurea o post-laurea nel $65 \%$ dei casi) e ha riconfermato le caratteristiche del welfare italiano, centrato sulla famiglia come ammortizzatore sociale e caregiver primario, in particolare grazie alle figure femminili. Durante l'emergenza, infatti, le famiglie non hanno potuto contare sul sostegno delle reti consuete come nidi e scuole, poiché chiusi, e nemmeno su nonne e nonni, poiché più a rischio di contagio; anche il ricorso a babysitter o assistenti a persone anziane o non autosufficienti è risultato poco sfruttato (dichiarato dal $5 \%$ degli intervistati).

I genitori che si sono occupati da soli dei figli (specie se di età inferiore ai 6 anni) hanno raccontato di aver avuto difficoltà a concentrarsi sul lavoro a causa di pensieri riguardanti la cura. I lavoratori autonomi hanno mostrano una maggiore soddisfazione nella conciliazione tra lavoro e famiglia rispetto ai dipendenti.

Un elemento che risulta aver favorito la gestione dello stress e delle fatiche è la collaborazione all'interno della coppia, che ha permesso una nuova divisione dei compiti di cura; collaborazione che tuttavia è emersa soprattutto tra le coppie giovani e meno in quelle in cui le donne over 50 si sono trovate spesso ad affrontare anche la cura dei genitori anziani o di familiari disabili o non autosufficienti.

La ricerca ha sondato anche quale sia stata la priorità tra lavoro e famiglia, rilevando profonde differenze sociali: ha attribuito più importanza al lavoro chi non ha figli o chi non si occupa direttamente della loro gestione, chi ha un titolo di studio e un reddito più elevato, mentre ha dato più importanza alla famiglia chi ha figli e se ne occupa personalmente, chi ha titolo di studio e reddito più basso, chi ha più familiari conviventi (Centro di Ateneo Studi e Ricerche sulla Famiglia, 2020). 
Nel gruppo di studiosi/e afferenti al Centro di Ricerche Educative su Infanzia e Famiglie (CREIF) del Dipartimento di Scienze dell'educazione di Bologna, tra fine marzo e fine aprile 2020, è stato diffuso attraverso il web il questionario Essere genitori ai tempi del Covid-19: disagi, bisogni, risorse, compilato da circa 800 genitori di figli/e in età compresa fra 3 e 18 anni (Gigli, a cura di, 2020; 2021). Il 41\% del campione ha tra 31 e 40 anni, il $40 \%$ tra 41 e 50 anni, il 5.6\% ha meno di 30 anni, e meno dell' $1 \%$ più di 60.

Per il $90 \%$ si tratta di madri, per il 10\% di padri; il loro livello di istruzione è piuttosto alto: il 58\% è laureato, il 34\% diplomato; il 74\% è lavoratore dipendente (di cui il $56 \%$ a tempo pieno e il $18 \%$ a tempo parziale); solo il 14\% è libero professionista e il $7 \%$ disoccupato/a.

Riguardo alla situazione lavorativa nell'emergenza da Covid-19 risulta che il 58\% lavora da casa, il 7\% si reca al lavoro come prima, il 6\% lavora fuori casa a orario ridotto; il $3 \%$ asserisce di aver perso il lavoro.

I partecipanti alla rilevazione risiedono per il $72 \%$ nel Nord Italia, $10.6 \%$ al Centro; meno dell' $1 \%$ in tutte le altre Regioni. Complessivamente il $76 \%$ è coniugato o ufficialmente convivente.

Tra i numerosi dati emersi riportiamo quanto affermato dai rispondenti alla richiesta di esprimersi in merito alle difficoltà vissute dal nucleo familiare: le maggiori difficoltà risultano risiedere nella conciliazione degli impegni professionali con quelli familiari, nella gestione degli aspetti emotivi e della vita scolastica dei figli, mentre risultano vissuti con un minore grado di difficoltà gli aspetti organizzativi, quelli economici, la cura di parenti anziani o malati (Ibidem).

Certamente ogni nucleo familiare si caratterizza come soggetto a sé ed elabora modalità di funzionamento proprie, senza seguire regole predeterminate così come ogni figlia/figlio nelle sue caratteristiche individuali e legate alla fase di crescita possiede risorse o presenta problematicità peculiari. D'altra parte, il confinamento, la chiusura delle scuole, la sospensione delle attività sportive e ricreative, l'assenza di contatti sociali non può che produrre un impatto forte e specifico sulla vita familiare e su quella dei figli, soprattutto in età scolare. Vale la pena anche solo menzionare come da una ricerca effettuata da Telefono Azzurro durante e a seguito del lockdown emerga un preoccupante aumento delle richieste di aiuto e dei tentativi di suicidio in età adolescenziale (Longo, 2021). Non potendo qui addentrarsi in una delle problematiche più gravi implicate nell'emergenza, possiamo ancora menzionare difficoltà specifiche - indagate, tra altre, da una ricerca attuata a livello nazionale dalla Società Italiana di Ricerca e Didattica - legate alla mancanza, carenza, 
o inadeguatezza e malfunzionamento degli strumenti tecnologici disponibili nel contesto domestico (necessari a più persone in caso di fratrie e allo smart working dei genitori). Per fruire della didattica online tutto deve funzionare: microfoni, telecamere, software. Si pensi alla necessità di un'efficiente connessione internet laddove la mancanza di linea o lo scarso segnale rendono impossibile partecipare a qualsiasi attività online. Si pensi ancora alle possibili difficoltà, almeno inziali, a utilizzare in maniera efficace i dispositivi, ad accedere alle piattaforme online, a comunicare mediante una chat dal pc, e altro ancora (Batini et. al., 2020; Lucisano, 2020).

Interessante e imprescindibile allora è poter entrare, seppur parzialmente, nel punto di vista degli adolescenti in merito a questa esperienza. Questo contributo cerca di farlo, portando ascolto e attenzione ai racconti e alle rappresentazioni espresse nella forma di brevi componimenti scritti da alunne/i di scuola secondaria di primo grado di Bologna, riguardo alla loro vita familiare nel periodo di confinamento della primavera del 2020.

\section{Una opportunità di ricerca}

L'opportunità di ricerca e riflessione nasce dalla disponibilità di un insegnante di Italiano e Storia, il professor Stefano Camasta ${ }^{2}$, che ha reso fruibili alla lettura e all'analisi gli scritti di ragazze e ragazzi di classi $2^{a}$ e $3^{\text {a }}$ della scuola secondaria di I grado "L.C. Farini" di Bologna (Istituto Comprensivo 12). Alunne a alunni sono stati coinvolti dall'insegnante nell'iniziativa lanciata dall'Editrice Pendragon, il 10 marzo 2020, e così presentata dalla stessa: «scrivi e pubblica sulla pagina Facebook Edizioni Pendragon un racconto inedito che tragga spunto dall'emergenza che stiamo vivendo. I cento più belli, a insindacabile giudizio della casa editrice, entreranno a far parte di un'antologia»?

${ }^{2} \mathrm{Al}$ professor Stefano Camasta va il mio apprezzamento per aver coinvolto le/gli alunne/i delle sue classi nell'occasione offerta da Pendragon, nonché un ringraziamento per avermi dato accesso alla lettura, all'analisi e alla citazione dei testi prodotti dalle/ dagli alunne/i (d'ora in poi, laddove non diversamente specificato, le note a piè di pagina si intendono a cura dell' Autrice, N.d.R.).

${ }^{3}$ La lunghezza massima per gli elaborati è stata indicata in 1800 battute e il termine per postarli fissato tra l'11 marzo e il 3 aprile 2020. Le proposte dovevano essere pubblicate sulla pagina Facebook delle Edizioni Pendragon. 
L'Antologiaiostoacasa (Aa.Vv., 2020), che di fatto ha selezionato 110 contributi tra gli 881 complessivamente ricevuti. Questa proposta letteraria nazionale offre un esempio delle diverse iniziative attivate durante le chiusure e i blocchi che tutti hanno coinvolto e condizionato tra la fine dell'inverno e la primavera 2020, e che a tutt'oggi costituiscono ancora, in larga parte, una realtà.

Il fatto che l'iniziativa sia stata accolta da un professore sensibile e attento alle risorse provenienti dal contesto extrascolastico (apertura peraltro riscontrabile anche in tempi di ordinaria vita scolastica), costituisce una risposta alla repentina necessità di ripensamento e rimodulazione della didattica e delle modalità di insegnamento-apprendimento nel virtuale, nella distanza fisica tra docenti e studenti. Nel pieno della pandemia, sono nate anche idee e inedite possibilità e, come nel caso qui considerato, nuove opportunità per contenere l'impatto della sospensione delle attività didattiche in presenza. Pur se all'interno di una circostanza drammatica che ha costretto all'interruzione improvvisa della partecipazione a un contesto socio-educativo fondamentale si sono aperte possibilità inedite per studentesse/studenti: ovvero, mettere a frutto le capacità d'uso delle tecnologie per scrivere testi, consultare la pagina Facebook dell'editore, esercitarsi nell'ideazione e nella produzione del testo scritto; leggere, conoscere e condividere gli scritti con i compagni/e e, non ultimo, esprimere i propri vissuti, in una fase difficile della vita individuale, familiare, collettiva, nonché scolastica.

Occorre tener presente che gli scritti sono stati prodotti e raccolti in risposta a una consegna assegnata da un professore e dunque non possono essere considerati espressione totalmente libera dall'influenza e dai condizionamenti di una possibile valutazione. Inoltre, fatta eccezione per quello che emerge dai componimenti, non si conoscono elementi delle vite personali e familiari degli/delle autori/autrici, della disponibilità in casa di strumentazioni e competenze digitali, dell'accesso più o meno agevole alla rete; delle loro abitazioni più o meno confortevoli in fase di confinamento, del numero di componenti dei nuclei familiari e delle relazioni tra loro, del coinvolgimento più o meno diretto nelle vicende di malattia e morte legate al Covid-19. Non è noto nemmeno il ruolo che i genitori di questi ragazzi/e hanno avuto nelle attività didattiche dei figli e dunque non si può escludere che alcuni degli scritti possano aver ricevuto input, revisioni, arricchimenti, orientamenti o altro da parte degli stessi genitori o di altri membri della famiglia. Ancora, non è noto se gli scritti che appaiono più realistici e meno frutto di fantasia ed invenzione riportino effettivamente vicende e temi legati alle esperienze e alle vite reali. 
Ciononostante, possiamo ritenere che i brevi elaborati di ragazze e ragazzi corrispondano all'aver messo a frutto la disponibilità di una via aperta per esprimere contenuti urgenti o almeno significativi per loro: adolescenti della città di Bologna che si sono trovati immersi in un contesto di vita trasformatosi profondamente e all'improvviso, nientemeno che per effetto di una pandemia (Lorenzini, 2020).

\section{I partecipanti}

Ragazzi/e, di età compresa tra 12 e 14 anni, sono stati chiamati a esprimere in poche parole scritte contenuti, pensieri, emozioni, paure e desideri collegati alla complessa esperienza in corso. Nella secondaria di I grado "L.C. Farini" di Bologna, in una classe $2^{\mathrm{a}} \mathrm{e}$ in una classe $3^{\mathrm{a}}$, sono stati raccolti dal professore di Lettere 32 brevi componimenti scritti, sviluppati da 33 alunne/i (uno scritto è stato realizzato da due compagne di $2^{a}$, insieme).

Nel dettaglio: 17 alunne/i ( 9 maschi e 8 femmine) appartengono alla classe $2^{\mathrm{a}}$ e 16 alunne/i ( 9 maschi e 7 femmine) alla $3^{\mathrm{a}}$. Non tutte/i le/gli alunne/i delle classi coinvolte hanno corrisposto alla consegna: ciascuna delle classi è infatti composta da 25 studenti (15 maschi e 10 femmine nella $2^{\mathrm{a}}$ e 13 maschi e 12 femmine nella $3^{\mathrm{a}}$ ). Non si conoscono le ragioni della mancata partecipazione di alcune/i alunne/i all'attività di scrittura: è possibile soltanto ipotizzare che possa essersi trattato di negligenza, oppure della conseguenza della difficoltà per alcuni a seguire la didattica online, o di altre motivazioni ancora. Risulta però che 17 alunne/i non hanno formulato gli scritti.

Per garantire l'anonimato delle/degli autrici/autori dei testi non è indicata la lettera assegnata alle classi (quella che ogni plesso scolastico utilizza per distinguere le sezioni) e quando verranno citati stralci degli scritti, questi saranno seguiti da una sigla, ad esempio: ( $1 \mathrm{f} 2^{\mathrm{a}}$ media) a indicare il numero progressivo attribuito alla/o scrivente, se si tratta di femmina o maschio $(\mathrm{f} / \mathrm{m})$, e se appartiene alla classe $2^{\mathrm{a}} \mathrm{O} 3^{\mathrm{a}}$.

\section{I principali nuclei tematici emersi dall'analisi dei componimenti}

Come detto, possiamo ritenere che i contenuti posti al centro dei componimenti - brevi, ma spesso più estesi di quanto richiesto da Pendragon (1800 caratteri) - possano condurci ai pensieri, desideri, bisogni, 
paure, aspettative, risorse di queste/i alunne/i, in fase più o meno avanzata dell'adolescenza. Numerosi temi ripercorrono i leitmotiv circolanti nel periodo considerato e che si sono imposti all'attenzione delle ragazze e dei ragazzi che li hanno narrati, commentati, in qualche caso rivisitati criticamente o rielaborati nella fantasia. Un terreno ampio, ricco ma anche pieno di incognite si apre all'interpretazione di contenuti, verso i quali possiamo in primo luogo, in senso pedagogico, porci in ascolto, con l'attenzione volta a dar voce e valore a un'importante possibilità di espressione, per poi cercare di comprendere e dunque ricavarne indicazioni per la riflessione e l'intervento educativo.

Dalla lettura dei 32 componimenti si coglie la presenza di scritti che traspongono contenuti dell'attualità nell'invenzione di storie fantastiche che sembrano riprendere temi dalla letteratura, da film di avventura, da videogiochi; la presenza di altri che ricalcano e descrivono uno o più aspetti della vita in corso; e di altri ancora che mostrano una compresenza di realtà e fantasia difficilmente districabile.

La maggior parte dei testi è scritta in prima persona; altri invece in terza, forse, a evidenziare il distanziamento come un bisogno. Bisogno di mantenere distanze, sul piano fisico quale motivo imperante nella fase pandemica, e in senso ampio quale necessità di proteggersi da un'esperienza complessivamente difficile e avvertita come fondamentalmente minacciosa. Altri componimenti ancora sono scanditi in giornate e orari, formulati in forma di diario.

Di cosa parlano, dunque, gli scritti? Indicherò in corsivo, supportandoli con la citazione di stralci tratti dai componimenti, alcuni dei temi ricorrenti e/o più significativi. Dall'analisi dei contenuti si evidenzia, anzitutto, la molteplicità dei temi toccati dalle ragazze e dai ragazzi. Ampia la gamma delle emozioni che si trovano espresse: a volte solo menzionate, altre argomentate, pur se nello spazio ridotto del componimento, e riferite a se stessi o ai membri della famiglia, agli adulti più vicini, ai coetanei, in qualche caso all'intera umanità.

In un caso singolare, emozioni e straniamento sono attribuite persino al plurinominato e sempre sottinteso coronavirus! Spesso, il virus è descritto tra distruttività e valore salvifico: il suo potenziale gravemente negativo pare frequentemente sotteso alle parole dei ragazzi: «il virus aveva attaccato con ferocia tutto il globo, alla fine l'unico superstite ero io» (2 $\mathrm{f} 2^{\mathrm{a}}$ media); «quest' anno inizia veramente benissimo, mio caro diario: volevo informarti che stiamo per morire tutti» $\left(9 \mathrm{~m} 2^{\mathrm{a}}\right.$ media); «...Quando vedo i dottori lavorare giorno e notte per salvare altre vite rischiando la loro, capisco quanto sia grave la situazione» ( $5 \mathrm{f} 2^{\mathrm{a}}$ media). 
D'altro canto, non mancano i richiami (presenti nei discorsi di molti, tra il senso comune e le riflessioni di studiosi che a vario titolo hanno commentato le vicende in corso) all'opportunità di trasformare una realtà drammatica in crescita personale e sociale, capace di potenziare il riconoscimento delle cose davvero importanti nella vita: solidarietà, empatia, rispetto dell'ambiente ecc.: «il clima, l'ambiente, gli animali lo ringrazieranno perché è diminuito l'inquinamento sia nell'atmosfera che nell'acqua» ( $4 \mathrm{f} 2^{\mathrm{a}}$ media).

La pandemia è soprattutto riflessione... È come se il tempo si fosse fermato perché si è stancato di correre e affannarsi dietro gli esseri umani che non fanno altro che sbagliare e non apprezzare le cose che hanno, continuando a dare tutto per scontato! Questa pandemia non conosce né ricco né povero, né giovane né vecchio, né malato né sano... Colpisce senza distinzione e io ho paura... (13 f $2^{\mathrm{a}}$ media).

Alcuni scriventi esplicitano come non avessero mai udito prima e non conoscessero il significato delle parole "virus" o "pandemia", scoprendo, poi, che corrispondono a privazione, solitudine, tristezza: «la TV, i giornali, i social dicono che è scoppiata la pandemia... Cos'è??? Mi sono chiesta... Sinceramente non sapevo neanche cosa fosse nello specifico... Anche se il nome non faceva sperare niente di buono! La pandemia è privazione!!! La pandemia è solitudine. La pandemia è tristezza...» (13 f $2^{\mathrm{a}}$ media). Il virus, spesso definito mostro o mostriciattolo o un Reale di nome ma non di fatto! è anche «un nemico invisibile che sta attaccando l'Italia, un virus che sta facendo preoccupare milioni di Italiani» $\left(13{\mathrm{~m} 3^{\mathrm{a}}}^{\mathrm{a}}\right.$ media); «IL CORONA NON PERDONA» (11 m $3^{a}$ media); «La causa di tutto è il famoso virus che nonostante porti la corona... Non regala niente di buono!» ( $13 \mathrm{f} 2^{a}$ media).

In molti scritti emergono incredulità, desolazione, preoccupazione, tristezza, paura, disorientamento per l'irrompere di nuovi modi di vivere: «le giornate erano tristi, senza rumori, in strada non circolava nessuna macchina» (6 $\mathrm{m} 2^{\mathrm{a}}$ media); «erano delle bellissime giornate, piene di sole, i fiori sbocciavano, ma non c'era nessuno fuori, nessuno correva, nessuno giocava...» (11 $\mathrm{m} 2^{a}$ media). La città è silenziosa, desolata; il mondo circostante ha perso la vita insita nel cinguettare degli uccelli, nell'abbaiare dei cani e nel miagolare dei gatti, nel vociare delle persone, nelle risa dei bambini.

Altamente ridotto è il rapporto con la natura e la possibilità di fare attività motoria ma il senso di perdita riguarda anche il rumore delle auto, 
le saracinesche abbassate. L'immobilità circostante provoca «un senso di angoscia e di paura» ( $13 \mathrm{~m}^{\mathrm{a}}$ media $)$, ma al contempo il cielo è più azzurro, l'aria tersa. Le lezioni sono soltanto online mediate e consentite da uno schermo e dal collegamento a internet, il lavoro dei genitori è quasi sempre in smart working.

La quotidianità di cui ragazze e ragazzi parlano è condizionata da una sovraesposizione mediatica, da telegiornali e "onnipresenti" notizie:

Ogni volta che lei guardava la televisione, in ogni canale andasse dove c'era il telegiornale si parlava solamente del coronavirus e dell'aumento di contagiati; quando si guardava un film e c'era la pubblicità si arrivava a parlare del coronavirus, su come lavarsi le mani, sul non avere contatti... Lei era stanca di sentire sempre le stesse cose... ( $12 \mathrm{f} 3^{a}$ media).

Le giornate scorrono al ritmo di parole inquietanti: «ascolto e osservo i genitori e la TV che parlano preoccupati di crisi economica, ricerca scientifica, caduta dei mercati e delle borse mondiali, di guerre senza le armi ma che comunque fanno del male» ( $4 \mathrm{f} 2^{\mathrm{a}}$ media).

Parole pronunciate dagli adulti, dai coetanei, dai media e da notizie affidabili e altre ingannevoli che, con la loro pervasiva presenza, si affastellano e si confondono. E confondono, rendendo improbabile qualsiasi fondata aspettativa o previsione. Non di rado infatti è dichiarata l'incapacità di capire e affrontare quanto sta accadendo:

Io sono un comune ragazzo di 14 anni e non so come affrontare questo brutto periodo visto che non ho mai avuto un'esperienza del genere. Un periodo in cui le scuole rimangono chiuse per un mese facendo delle videolezioni, un mese di sofferenza senza riuscire a vedere i propri amici e stando in casa tutto il giorno, una cosa impossibile per me. Infatti un giorno sono andato a giocare a pallavolo con mia sorella in un parco sotto casa mia e la polizia ci ha fermato di-

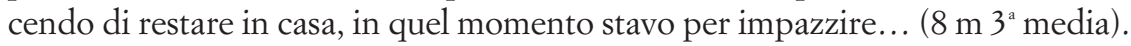

Sono esplicitati lo stress e la sofferenza della quarantena, dunque ne ricaviamo su un piano educativo -, il bisogno di essere sostenuti e accompagnati nel comprendere e affrontare. È esplicitata «paura del presente e paura di non arrivare al mio domani... paura di non riuscire a realizzare delle ragazze e dei ragazzi compare anche nel dichiarare un (reale o di fantasia) coinvolgimento personale e familiare nella malattia. E nelle sue conseguenze più gravi.

Sono sottolineati il peso e la difficoltà di accettare le rinunce: in particolare il non poter vedere gli amici. 
Notevole rilevanza è assegnata negli scritti al tema delle regole. Regole eteroimposte da rispettare pena la diffusione del contagio, sino alle possibili estreme conseguenze della perdita della vita propria e altrui. Molte volte se ne richiama la necessità del rispetto: «dopo la videolezione un suo amico gli chiede se vuole uscire e Jack risponde: "è nostro dovere restare a casa perché ognuno di noi deve fare la sua parte" $\gg(9 \mathrm{~m}$ $3^{\mathrm{a}}$ media). Altre volte, la realtà della trasgressione:

La situazione italiana è tra le peggiori, perché secondo me abbiamo sottovalutato la cosa all'inizio e ora ne paghiamo le conseguenze, avendo 200 nuovi casi ogni 24 ore. Io credo che ci siano persone talmente poco responsabili che, nonostante la situazione, continuano a uscire nei parchi o ad andare sotto casa di qualcuno per non farsi beccare dalla polizia. [...] Dobbiamo sentirci in dovere di rispettare le regole perché potrebbe ammalarsi ciascuno di noi. Siamo in un periodo di quarantena per evitare l'aumento del contagio, anche se non sta funzionando molto finché la gente continua ad uscire per ragioni non essenziali... ( $5 \mathrm{f} 2^{\mathrm{a}}$ media).

È da notare come in diversi racconti, in prima o terza persona, emerga un forte senso di colpa conseguente al non avere rispettato le regole, cosa che talvolta porta con sé conseguenze gravissime e fortemente punitive: la propria malattia, la morte della propria madre, del nonno ecc. di tutti.

Ancora una volta Jack sta uscendo con i suoi amici nonostante la quarantena obbligata, ma ormai era ora di andare a casa. Appena arrivato va subito a letto dato che deve ancora fare i compiti e si dovrà svegliare presto domani. Lui sta aprendo gli occhi quando si accorge di essere in casa da solo, si alza da letto e va in cucina dove trova un biglietto con scritto che sua mamma si è ammalata e dovrà restare in ospedale. Jack sa che se è in casa da solo nessuno può dirgli di partecipare alle videolezioni, quindi si siede sul divano a guardare la televisione. Il giorno dopo continua a non fare le videolezioni e fa così per altri due giorni, poi inizia a preoccuparsi e riflette che non doveva uscire di casa perché magari è stato lui la causa della possibile morte dei suoi genitori. Sotto casa sua ci sono

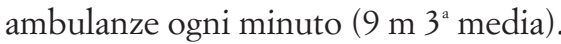

Dopo una settimana il mio amico mi ha chiamato dicendomi che sua nonna era all'ospedale perché positiva al tampone e che a breve sarebbe morta perché aveva già diverse patologie e la sua vita era attaccata a un respiratore. In quel momento mi crollò addosso il mondo, mi sentivo estremamente in colpa perché io ripensandoci avevo proprio quei sintomi ma non ero rimasto nella mia casa anzi avevo contaminato una donna innocente che come unica colpa aveva quella di avere le difese immunitarie troppo basse. Il giorno dopo andai all'ospedale

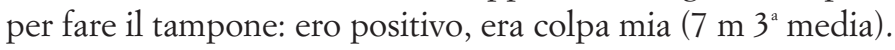


La morte, la sua presenza, la sua assenza, la riduzione della sua gravità si collocano in modo esplicito, oppure sullo sfondo, di gran parte degli scritti. Tuttavia, speranza, fiducia e proiezione nel futuro emergono, anch'esse, con forza: le/gli autrici/autori confidano nell'esito positivo di una vicenda difficile, nella possibilità di contare sulle proprie forze, nei buoni risvolti dell'osservare le regole, nei risultati dell'impegno di medici e infermieri e della ricerca scientifica.

Con piacere ed emozione sono menzionate le occasioni di condivisione vissute quando in diverse zone della città di Bologna cittadini collegati tra loro attraverso i social network o grazie agli scambi di vicinato da un terrazzo all'altro, si sono organizzati per ritrovarsi, suonando e cantando insieme sul far della sera proprio sui balconi delle abitazioni, vivendo momenti di socialità e solidarietà di contrasto al distanziamento e di sostegno a sentimenti di appartenenza a una comunità che, se unita, può sentirsi ed essere più forte: in questi ultimi giorni è stato coniato il motto \#iorestoacasa, milioni di Italiani hanno appeso ai balconi una bandiera con l'arcobaleno e con su scritto "andrà tutto bene" $\left(13 \mathrm{~m} \mathrm{3}^{\mathrm{a}}\right.$ media); e ancora:

bisogna essere forti e resistere allo stress psicologico restando uniti; ho avuto le prove che le persone fossero unite una sera quando su un social è girata la voce di applaudire fuori dalla finestra: nella mia via si sentivano molte persone. Lo so che è una piccola cosa, ma sono proprio tante piccole cose che ci rendono più forti $\left(8 \mathrm{~m} 3^{\mathrm{a}}\right.$ media $)$.

Tra le emozioni positive va certamente ricordata l'iniziale euforia per la chiusura delle scuole: «notiziona! Ho sentito dire che si starà a casa altre tre settimane e si continuerà con le videoconferenze, fantastico no?» $\left(9 \mathrm{~m} 2^{\mathrm{a}}\right.$ media $)$.

Suona la sveglia, mi alzo sono le sette del mattino oggi è una giornata come altre, fuori c'è il sole e i miei genitori sono ancora nel letto. Vado in cucina per fare colazione, prendo il mio telefono e vedo che nel gruppo della classe c'è una grande gioia, tutti esultano e sembrano felici mentre io non capisco tutta questa felicità, allora chiedo al mio migliore amico che cosa fosse successo e lui mi dice che le scuole di tutta Italia resteranno chiuse fino a data da destinarsi a causa del coronavirus. Io inizialmente non capisco perché non sapevo neanche cosa fosse questo virus $[\ldots]$ ( $7 \mathrm{~m}^{3 \mathrm{a}}$ media $)$.

La gioia iniziale risulterà, però, ben presto scemare, lasciando posto alla noia e anche al desiderio per la riapertura delle scuole stesse: «io sono qui in casa a provare a divertirmi da solo ma spero che finisca e che 
riaprano tutto, sì anche la scuola perché mi mancano i miei compagni di classe. Con un abbraccio...» (16 $\mathrm{m} 3^{\mathrm{a}}$ media).

La noia, che per questi adolescenti si rivela potente, persino tormentante, è menzionata numerose volte e spesso collegata all'assenza degli

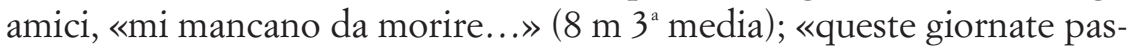
sano molto lentamente e sono super noiose...» (15a e $15 \mathrm{~b} \mathrm{f} 2^{\mathrm{a}}$ media). "Per fortuna" ci sono il cellulare, il virtuale, il potere di internet, che consentono di seguire lezioni e vedere i compagni pur se in uno schermo: gli amici (e dopo i parenti) sono nella mente, nel cuore... E nello schermo. La fantasia e l'invenzione aiutano a fronteggiare la noia. Alla «noia nei suoi occhi» ( $12 \mathrm{f} 3^{a}$ media), alle «giornate tristi, senza rumori...» (6 $\mathrm{m} 2^{\mathrm{a}}$ media), al «mi sto annoiando un sacco...» $\left(8 \mathrm{~m} 2^{\mathrm{a}}\right.$ media) qualcuno non trova soluzioni o almeno non le esprime negli scritti: «ora hanno praticamente vietato di uscire di casa, è una vera rottura» $\left(9 \mathrm{~m} 2^{\mathrm{a}}\right.$ media); «... Si impegna per fare i compiti nella mezzora prima della videolezione e alle nove è nella videolezione più noiosa della storia» $\left(9 \mathrm{~m} 3^{\mathrm{a}}\right.$ media).

Altri scoprono la via della creatività, spesso, grazie agli amici: «ieri pomeriggio mi stavo annoiando, allora ho chiamato il mio amico e insieme abbiamo inventato un nuovo gioco elettronico che abbiamo chiamato New Pandemic!» (3 m 2a media).

Infine, per far fronte alla noia alcuni menzionano l'intervento dei genitori.

\section{La centralità della vita familiare e del ruolo genitoriale}

Nei componimenti, i temi relativi ad amiche e amici, ai sentimenti di mancanza per la loro assenza fisica, ma al tempo stesso la loro significativa presenza nelle relazioni mantenute nei pensieri e nelle fantasie, e grazie agli indispensabili e onnipresenti strumenti tecnologici, non passano mai in secondo piano.

Ciononostante, non manca né sorprende la rilevanza della vita in famiglia, espressa attraverso tematiche ed emozioni diverse, collegate ai diversi contesti familiari, alle loro risorse e problematiche nuove o pregresse. Troviamo scritti (la minoranza, in verità) che appaiono carichi di disagio sul piano emotivo: «l'unica cosa importante da evitare in questo periodo è impazzire e mantenere la calma psicologicamente, pensando che tutto passerà presto e pensando a tutte le persone che ci mancano» $\left(8 \mathrm{~m} \mathrm{3}^{\mathrm{a}}\right.$ media). Affermazioni forti, che lasciano intendere come il disagio scaturisca proprio dalle relazioni familiari, meritano attenzione, ascolto, 
presa in carico: «in casa non faccio altro che litigare con mia madre e mia sorella, però per fortuna riesco a tranquillizzarmi chiamando i miei amici al telefono» ( $\mathrm{m} 83^{\mathrm{a}}$ media).

Le tensioni collegate alla complessiva condizione di emergenza penetrata nella vita quotidiana possono rendere più aspri i conflitti dell'età adolescenziale. Lotte e contrapposizioni andrebbero, però, tradotte in discordanze di pensieri e sentimenti da ritenersi legittimi, da tollerare e gestire. Reggere il conflitto, contrastandone la distruttività, può essere particolarmente difficile, e dunque ancora più importante, quanto più le modalità per esprimerlo, da parte dei figli, assumono toni polemici e aggressivi (Contini, 2001, in Milani, a cura di). Certamente si tratta di un compito non banale e nemmeno "naturale", che, anzi, ci ricorda anche la necessità dei genitori di essere sostenuti nel proprio ruolo educativo, al fine di salvaguardare il benessere di tutti i membri di una famiglia (Milani, 2001, a cura di).

D'altra parte, in diverse testimonianze la vita familiare e il ruolo genitoriale sono descritti come capaci di offrire un determinante sostegno al vivere una situazione del tutto sconosciuta, al sopportare la perdita di buona parte della vita precedente all'esplodere della pandemia, a trasformare la noia in opportunità:

i ragazzi potevano giocare insieme perché le loro stanze erano collegate e per prima cosa la madre gli disse di fare un disegno su questo virus. Dopo qualche settimana, Sophie e George iniziavano ad annoiarsi e lo dissero ai genitori, così il padre si fece venire in mente qualcosa da fare tutti insieme, allora iniziarono a fare lavoretti e giochi inventati ( $10 \mathrm{f} 2^{\mathrm{a}}$ media).

Di segno particolarmente positivo i racconti dai quali emerge ciò che potremmo definire la "bellezza della quarantena":

Durante la quarantena ho fatto tante attività, man mano che passavano i giorni trovavo sempre qualcosa di nuovo da fare per passarmi il tempo e non annoiarmi. Inizialmente facevo i compiti, guardavo la TV e passavo dal divano al letto annoiata, in seguito visto che anche gli altri componenti della mia famiglia sono stati costretti a rimanere a casa dal lavoro o da scuola ci siamo cimentati in altre attività ( $10 \mathrm{f} 3^{\mathrm{a}}$ media).

Da tale avvio, nello scritto di questa giovane di $3^{a}$ media, nasce un elenco, lungo e soddisfatto, di modi utili e divertenti per non annoiarsi e soprattutto per essere insieme: fare dolci di tutti i tipi con la mamma e poi ginnastica e cyclette, gli esercizi su YouTube con addominali, flessioni 
e salti con la corda in terrazza, dove si poteva anche prendere il sole. Coltivare la passione per il disegno. Aiutare il padre a mettere ordine nel garage, smontare, pulire, verniciare di verde acqua e rimontare una vecchia bici con un «risultato fantastico». Insieme alla sorella provarsi tante tonalità di smalto e di trucco, farsi fare i boccoli; vedere film e serie TV, mangiando popcorn; giocare a pallavolo, a calcio in garage (per non incontrare nessuno), alla X-box, a Fifa o Just dance. Riunirsi tutti dopo cena e giocare a Risiko o a carte, trasformare il tavolo in un campo da ping pong, dove giocare gli uni contro gli altri, divertendosi molto. E, ancora, guardare vecchie fotografie, sistemare gli armadi di tutti, ridendo nello scoprire i vestiti dei genitori da giovani: «volevano buttarli quindi io ho deciso di cucirli e creare dei vestiti per il cane del nostro vicino, con il quale giocavamo spesso quando non eravamo rinchiusi» (10 f $3^{a}$ media). Fare videochiamate con la nonna, per rimanere in contatto, passare il tempo, farsi dare la sua ricetta segreta e fare i tortellini «venuti buonissimi!!!» (Ibidem).

$\mathrm{Ma}$, più di tutto in questa diversa e speciale normalità nell'organizzazione delle giornate: «La cosa positiva di questa quarantena è che posso passare tanto tempo con la mia famiglia, possiamo stare tutti insieme» ( $10 \mathrm{f} 3^{\mathrm{a}}$ media). La testimonianza racconta con vitale entusiasmo il passaggio dalla noia profonda al piacere delle attenzioni e della cura reciproca, al divertimento pieno e creativo del fare insieme, dell'ideare e realizzare attività condivise con i membri della famiglia o con alcuni tra loro, di volta in volta, a seconda dell'attività. Questo, in effetti, si mostra come una delle maggiori, inedite e forse irripetibili, opportunità offerte dalla quarantena, in un tempo liberato dal sovraccarico di impegni, in cui ci si trova - pur giocoforza - insieme. Un'opportunità per tutti coloro che ne hanno saputo, e potuto, beneficiare.

\section{Responsabilità genitoriali}

Chi si occupa di educazione non può non porre in luce come tutti i temi emersi dagli scritti di ragazze e ragazzi, nelle loro articolazioni positive o problematiche e nella loro densa dimensione emotiva, meritino ascolto, attenzione, valorizzazione, risposte.

Complessivamente, le famiglie sono state chiamate a far fronte con le risorse e le potenzialità già possedute o cercate, e sviluppate o meno in relazione al sopraggiungere di emergenze molteplici e all'emersione di nuovi bisogni, quali ad esempio quelli conoscitivi: «io volevo sapere e ca- 
pire tutto quello che stava accadendo» ( $1 \mathrm{f} 2^{\mathrm{a}}$ media). Bisogni conoscitivi che strettamente si intrecciano ad altri sul piano emotivo. Conoscere e capire aspetti scientifici della diffusione di un virus e dunque come proteggere sé stessi e gli altri, ad esempio, può aiutare anche ad affrontare e orientare adeguatamente ansie e paure e a interagire in modo efficace con la nuova situazione, cogliendo le occasioni positive effettivamente presenti in una fase complessa, evitando la minimizzazione e la negazione dei problemi, ma anche la loro esasperazione.

Gli adulti affettivamente e educativamente significativi per le/i più giovani si sono trovati a dover accogliere i loro vissuti di disorientamento, i nuovi dispiaceri e problemi; ad aiutarli a gestire le preoccupazioni, a sopportare le rinunce, a rispettare e comprendere il senso delle regole. Anzitutto, gli adulti in famiglia sono stati chiamati ad accorgersi di questi aspetti critici, sia quando espressi in modo esplicito, come nel caso che qui menziono:

Se mi avessero detto che sarebbe arrivato un giorno in cui il mondo si sarebbe fermato avrei sorriso, se mi avessero detto che un giorno non avrei più potuto abbracciare i miei cari... Avrei sorriso se mi avessero detto che avrei dovuto rinunciare alla mia più grande passione (il calcio) avrei sorriso pensando che per farmi smettere mi sarei dovuta solo spezzare una gamba! Se mi avessero detto che non sarei potuta andare a scuola... Beh... Forse sarei stata felice, ma avrei riso comunque pensando fosse una cosa impossibile! Oggi che tutto questo è la dura realtà avrei tanto voluto ridere... Ma mi viene solo da piangere ( $13 \mathrm{f} 2^{\mathrm{a}}$ media).

Sia quando aspetti critici non sono manifestati apertamente ma in forma velata, trasposta nella fantasia, da interpretare. Quando ad esempio gli scritti fanno ricorso all'invenzione ma risultano attraversati da intensi sentimenti di colpa, laddove gli autori si attribuiscono la responsabilità della morte dei propri genitori o della nonna di un amico ai quali avrebbero trasmesso la malattia, dopo aver trasgredito al confinamento, come negli stralci menzionati sopra di $\left(7 \mathrm{~m} 3^{\mathrm{a}}\right.$ media $)$ e $\left(9 \mathrm{~m} 3^{\mathrm{a}}\right.$ media).

Quando poi il virus assume le sembianze di "serpenti velenosi" che infestano una città, terrorizzano, aggrediscono e uccidono molti dei suoi abitanti, non emergono forse - oltre al senso di colpa conseguente a trasgressione delle regole e, pur in un finale sostanzialmente positivo pesanti sensazioni di pericolo e minaccia che gravano su chi scrive, su tutti e ciascuno?

La prima cosa che vidi appena arrivai a Charleston fu una folla di gente che correva con il terrore stampato in faccia. Stavano scappando, ma la vera 
domanda era: "Da chi? Da che cosa?". Mio padre abbassò il finestrino per chiedere informazioni, una signora farfugliò: "Serpenti ovunque... Scappate!!!". Ci dirigemmo verso la nostra nuova casa, a mia madre infatti era stato offerto un posto da medico primario in questa cittadina che non poteva rifiutare. Ovunque si sentivano delle urla, poco distante dal vialetto di casa giaceva un'anziana signora. Mia madre si avvicinò al quel corpo senza vita: notando punture sulla caviglia, pensò subito al morso di un serpente. Dopo aver chiamato la polizia, si recò all'ospedale dove un'infermiera le spiegò tutto: "inspiegabilmente, da qualche tempo la città è invasa dai serpenti che attaccano gli abitanti provocando morte e panico". Mia madre, incredula, chiamò il sindaco ed insieme decisero di far restare la popolazione a casa: si poteva uscire solo se strettamente necessario. Così anch'io mi ritrovai chiuso tra quattro mura. Inizialmente non me ne preoccupai, dato che sono sempre stato un tipo solitario: non sarebbe stato difficile restare a casa. Mia madre intanto era sempre in ospedale, lavorava ad un antidoto al veleno insieme agli infermieri e a qualche coraggioso volontario. Dopo un mese il pericolo era sempre presente, tuttavia non riuscivo più a resistere chiuso in casa! Di nascosto uscii, pensando che avrei fatto una semplice passeggiata, ma a un certo punto sentii una fitta alla gamba: ero stato attaccato da un piccolo serpente, sbucato dal nulla! Per fortuna Joe, il vicino, mi vide dal suo giardino e mi staccò il serpente di dosso; purtroppo però fu morso anche lui. A stento raggiungemmo l'auto per andare in ospedale. Mia madre e la sua squadra avevano appena prodotto in laboratorio l'antidoto, in quel momento però avevano una sola dose. Decisero che sarebbe toccata a me perché Jo, malato di Parkinson, aveva meno possibilità di farcela. Dopodiché persi i sensi. Quando mi risvegliai i rumori erano amplificati, i passi degli infermieri rimbombavano nel silenzio delle stanze d'ospedale. Invece di essere felice per essere vivo, fui colto da un'improvvisa angoscia, pensieri tristi iniziarono a comparire nella mia mente, uno dietro l'altro: "Possibile che non sia riuscito a stare a casa? Come potevo essere stato così egoista da mettere a rischio la mia salute e, soprattutto, come potevo essere responsabile della morte di Jo? Lui che mi aveva soccorso senza nemmeno conoscermi?”. Mi alzai stordito e andai verso il corridoio. A un certo punto vidi Jo, mi sembrava un miraggio, ma quello che io non sapevo è che mia madre era riuscita a preparare un'atra dose di antidoto giusto in tempo per salvarlo ( $3 \mathrm{f} 3^{\mathrm{a}}$ media).

La perdita della quotidianità conosciuta, dei rituali, del piacere di celebrare le ricorrenze fa pensare a cosa si sarebbe potuto fare prima ma ora è precluso. Questi pensieri si mescolano alla consapevolezza dei divieti e dei pericoli del presente e di cui è pieno il mondo fuori dal protetto ambiente domestico. Si esprimono nell'impossibilità di capire quello che sta accadendo come se fossimo piombati tutti in un surreale "manicomio": 
Mentre guardo l'ora sul pc aspettando che si avvii la lezione online, mi accorgo che è il 19 marzo 2020. È la festa del papà. Penso immediatamente che, terminata la lezione, sarei andato a comprargli un regalo, per cui la mia testa inizia a pensare a che sorpresa avrei potuto fargli. Ci sono: una cover per il cellulare. Adesso che ricordo ne ho vista una che sicuramente gli piacerà, in quel negozio in centro. Prendo l'autobus e in 20 minuti sono già tornato, comunque prima che lui rientri dal lavoro. Il piano è perfetto, devo solo attuarlo. Mi ricordo solo ora che mia madre è in casa, non mi farà mai uscire sapendo che in quel momento c'è la lezione, devo uscire di nascosto. Devo uscire dalla finestra. Una volta preso l'autobus mi accorgo che l'autista, insieme al passeggero accanto, ha sul collo tanti puntini verdi, devo scendere alla prossima fermata, così prenoto. Scendendo mi accorgo che quei due non erano gli unici ad avere puntini verdi. Così penso che dopo aver preso il regalo sarei tornato subito a casa per non aver a che fare con quella stranezza. Entro nel negozio, ma, appena varcata la soglia, il proprietario mi punta un fucile contro e mi ordina di andare via. A questo punto non so cosa fare, potrei provare a correre verso casa o... No, non trovo alternative, devo andare a casa. Iniziata la corsa comincio a vedere sfocato, così mi fermo per stropicciare gli occhi. La vista continua a offuscarsi finché non la perdo completamente e svengo. Apro gli occhi in una camera tutta bianca, vedo avvicinarsi un uomo che mi porta in una stanza piena di persone, persone non normali, persone... malate, malate di mente! Cerco un adulto (sano) per chiedere perché sono lì ma nessuno vuole darmi informazioni, non capirò mai perché sono finito in un manicomio (14 $\mathrm{m} 2^{\mathrm{a}}$ media).

Queste/i alunne/i hanno prodotto scritti interessanti e di piacevole lettura. La dimensione della fantasia che trasfigura la realtà oltre a mostrare il potenziale di un proficuo esercizio della creatività in forma scritta, sembra sostenere l'espressione di sentimenti di paura, preoccupazione, disagio. Verso questi dovrebbe attivarsi il ruolo adulto, affettivo ed educativo al contempo. Va, d'altro canto, considerata anche la difficoltà dei genitori ad affrontare con i figli e probabilmente anche con se stessi i risvolti più pesanti del presente, senza spaventarsi di fronte alla sua complessità anche nei suoi risvolti emotivi e dolorosi.

Ieri mentre guardavo beatamente la TV vedo arrivare mio padre nel salotto, inizialmente pensavo che volesse andare in cucina ma poi si dirige verso di me con aria triste e inizia uno di quei discorsi che fanno i genitori in cui girano intorno a una cosa per mezz'ora, ma per essere sinceri a me non interessava. Dopo un po' che sento la voce di mio padre come sottofondo nell'accozzaglia di parole riesco a carpire che tutto quel discorso era per dirmi che mia nonna era malata di coronavirus. Pensavo che stesse scherzando, ma poi si mise a piangere e allora capii che non scherzava affatto $\left(8 \mathrm{~m} 2^{\mathrm{a}}\right.$ media). 
Anche i professionisti di ambito pedagogico ed educativo - che pur hanno subito a propria volta l'impatto rilevante della pandemia - dovrebbero recuperare e ripensare la propria funzione e capacità di offrire ascolto e supporto alle famiglie e al ruolo genitoriale (Gigli, a cura di, 2020; 2021).

\section{Per concludere: qualche considerazione in senso educativo interculturale}

Comporre questi brevi scritti ha costituito per le/i giovani autori/ autrici l'opportunità di seguire una via aperta all'espressione di temi cogenti o quantomeno presenti nei loro pensieri. Possiamo ritenere si sia trattato anche di una occasione per far sì che la costrizione del distanziamento fisico non si traducesse in un distanziamento dalle proprie emozioni e vissuti.

Nell'età di queste/i giovani, se è indispensabile il confronto con i pari, resta forte la necessità di ascolto e rielaborazione condivisa con gli adulti affettivamente e educativamente significativi nella loro vita, in famiglia, a scuola. Per questo, ma anche per il grande interesse su piani interdisciplinari (dalla biologia alla letteratura, alla storia, dalle lingue straniere alla matematica, all'arte ecc.) che i contenuti emersi presentano, ritengo importante sottolineare come ben meriterebbero di essere ripresi e valorizzati in ambito scolastico. Messi a frutto anche per ri-significare l'esperienza vissuta nei mesi di sospensione connettendola al ritorno a una quotidianità che ancora oggi (febbraio/marzo 2021) è soggetta a forti limitazioni e resta differente da prima, quando il Covid-19 non era noto e, per molti, nemmeno il significato della parola e della realtà di una pandemia. Ripresi, condivisi e discussi in forma di conversazioni di gruppo in ambito scolastico (online o in presenza) possono aiutare ad avvicinarsi a nuovi apprendimenti, a dare nuova forma alle relazioni tra insegnanti e allieve/i, e tra allieve/i, a conferire senso e a mantenere continuità tra aspetti del tutto inediti nell'esperienza di ognuno e di tutti entro quella che è stata ed è una effettiva frattura nel percorso scolastico e di vita dei più giovani, e non solo.

Riprendendo il focus del nostro discorso, il contesto familiare - da sempre crocevia complesso e articolato di progetti di vita che reciprocamente si connettono e si influenzano (Contini, 2001, in Milani, a cura di) - risulta implicato in tutte le dimensioni dell'esperienza: cognitive, affettive, relazionali. Ne deriva un ruolo genitoriale chiamato, sul piano educativo e affettivo - come sempre lo è, ma ora in relazione a nuove esigenze e condizioni -, ad aver cura dei bisogni conoscitivi, emotivi, 
relazionali dei figli, oltre che dei propri in quanto adulti, soggetti singoli $\mathrm{e}$ in interazione in una coppia e in una famiglia.

Anche la vita familiare - pur se gravata da problematiche molteplici -, può e dovrebbe offrire occasioni per promuovere, con consapevolezza e intenzionalità, processi di conoscenza e riflessione centrati su rispetto e riconoscimento reciproco, nonché sul confronto critico, contribuendo alla costruzione di un nuovo sapere comune e nuove possibilità di relazione, che ognuno può portare con sé anche fuori dal contesto familiare. La difficoltà nel sopportare i sacrifici e le restrizioni può divenire opportunità per condividere e sviluppare il senso civico e la responsabilità del rispetto delle regole, proficua per sé e per gli altri. Trasformare in modo condiviso prassi e abitudini consolidate può intrecciarsi all'opportunità di favorire la conoscenza e la comprensione di quanto sta accadendo, ponendosi insieme domande: in quale situazione ci troviamo? Cos'è e come si comporta un virus? Quali sono le cause complesse, locali e globali, di una pandemia? Siamo tutti coinvolti in un mondo globalizzato, come possiamo agire per garantire il bene individuale e delle persone a noi più care, ma anche della collettività in senso ampio, sino ad abbracciare la dimensione del pianeta?

Una situazione difficile e dai risvolti drammatici offre occasioni per riflettere insieme sulle possibili risposte, per cercare informazioni e fonti attendibili anche sul piano scientifico. Occasione per comprendere che siamo immersi, che piaccia o no, che se ne colgano o meno i rischi e anche le risorse, in una dimensione di vita globalizzata, nella quale anche una pandemia dimostra il nostro essere interrelati e interdipendenti a livello planetario, cittadini di uno stesso mondo che è patria di tutti (Morin, Kern, 1993, trad. it. 1996) ed entro il quale molta parte dei nostri destini è comune.

Qui non posso che ricordare la fondamentale importanza di una Pedagogia e di una Educazione interculturale (Bolognesi, Lorenzini, 2017) anche all'interno della famiglia (Portera, 2004) per crescere e cambiare insieme in una situazione altamente problematica che, proprio nella sua complessità, impone e offre inedite opportunità, sollecita soluzioni creative, attivando risorse già possedute o cercate ex novo e maturate insieme.

\section{Riferimenti bibliografici}

Aa.Vv. (2020): \#antologiaiostoacasa. Racconti dal marzo 2020. Bologna: Pendragon. (https://www.youtube.com/watch?v=B7PYJRU5nhc\&feature=youtu. be; data di ultima consultazione: 20.1.21). 
Batini F., Barbisoni G., Pera E., Toti G., Sposetti P., Szpunar G., Gabrielli S., Stanzione I., Dalledonne Vandini C., Montefusco C., Santonicola M., Vegliante R., Morini A.L.; Scipione L. (2020, dicembre): Un modello di analisi delle domande aperte nell'indagine nazionale SIRD sulla didattica a distanza durante l'emergenza Covid-19. RicercAzione, 12(2), pp. 47-71.

Bolognesi I., Lorenzini S. (2017): Pedagogia interculturale. Razzismo, pregiudizi, impegno educativo. Bologna: Bononia University Press.

Centro di Ateneo di Studi e Ricerche sulla Famiglia (2020): Il lavoro da casa durante l'emergenza. L'impatto sulle famiglie (https://centridiateneo.unicatt. it/famiglia-ricerca-il-lavoro-da-casa-durante-1-emergenza-l-impatto-sullefamiglie; data di ultima consultazione: 27.1.21).

Contini M. (2001): La famiglia crocevia complesso di progettualità. In Milani P. (a cura di): Manuale di Educazione Familiare. Ricerca, intervento, formazione. Trento: Erickson.

Gigli A. (a cura di) (2020): Infanzia, famiglie, servizi educativi e scolastici nel Covid-19. Riflessioni pedagogiche sugli effetti del lockdown e della prima fase di riapertura giugno 2020. CENTRI.UNIBO.IT/CREIF. Bologna: Alma Mater Studiorum - Università di Bologna.

Gigli A. (a cura di) (2021): OLTRE L'EMERGENZA. Sguardi pedagogici su infanzia, famiglie, servizi educativi e scolastici nel Covid-19. Parma: Edizioni Junior-Bambini.

Lorenzini S. (2020): Marzo 2020. Il sentimento della perdita e la proiezione nel futuro nelle parole di alunne/i di scuola secondaria di primo grado di Bologna. "...e adesso scrivo le mie memorie su questo quadernino prima che la vita me lo possa negare". In Gigli A. (a cura di): Infanzia, famiglie, servizi educativi e scolastici nel Covid-19. Riflessioni pedagogiche sugli effetti del lockdown e della prima fase di riapertura giugno 2020, cit., pp. 92-100.

Longo G. (2021): Chiusi in casa, zero scuola e genitori ostili: boom di adolescenti che tentano il suicidio (https://www.lastampa.it/topnews/primopiano/2021/02/04/news/chiusi-in-casa-zero-scuola-e-genitori-ostiliboom-di-adolescenti-che-tentano-il-suicidio-1.39856996; data di ultima consultazione: 4.2.19).

Lucisano P. (2020): Fare ricerca con gli insegnanti. I primi risultati dell'indagine nazionale SIRD "Per un confronto sulle modalità di didattica a distanza adottate nelle scuole italiane nel periodo di emergenza COVID-19). Lifelong, Lifewide Learning (LLL), n. 36, pp. 3-25.

Marrocco A. (2020): Anna Oliverio Ferraris: "I ragazzi soffrono la sindrome della capanna. Non vanno confinati ma indirizzati (https://www.msn.com/it-it/ lifestyle/notizie/anna-oliverio-ferraris-i-ragazzi-soffrono-la-sindrome-dellacapanna-non-vanno-confinati-ma-indirizzati/ar-BB1b5SRW; data di ultima consultazione: 2.1.21).

Milani P. (a cura di) (2001): Manuale di Educazione Familiare. Ricerca, intervento, formazione. Trento: Erickson.

Morin E., Kern A.B. (1993): Terra-patria. Trad. it. Milano: Raffaello Cortina, 1996. Portera A. (2004): Educazione interculturale in famiglia. Brescia: La Scuola. 